\title{
artigo
}

\section{A usabilidade do teste molecular rápido para o diagnóstico da tuberculose em um município da amazônia ocidental}

The usability of the rapid molecular test for the diagnosis of tuberculosis in a west amazonian municipality La usabilidad de la prueba molecular rápida para el diagnóstico de tuberculosis en un municipio de la amazónica occidental

\section{RESUMO}

Objetivo: Descrever a usabilidade do Teste Molecular Rápido para a Tuberculose (TMR-TB) em Porto Velho, Rondônia. Método: Estudo descritivo, transversal e quantitativo, desenvolvido a partir dos registros dos doentes de tuberculose notificados entre janeiro a agosto de 2017 e que realizaram o TMR-TB. Os dados foram analisados por meio de estatística descritiva, após atender aos preceitos éticos. Resultado: Foram analisadas 2.317 amostras, solicitado principalmente pelo complexo penitenciário $(33,1 \%)$, maior quantidade de resultado positivo na Atenção Terciária $(27,8 \%)$, maior número de solicitações em maio $(19,6 \%)$ e resultado positivo em junho $(17,1 \%)$. A amostra mais analisada foi o escarro $(97,6 \%)$ que representou $100 \%$ dos resultados positivos. 0 tempo médio entre o diagnóstico e início do tratamento foi 3,54 dias (dp= $\pm 8,32$ ), e forma pulmonar predominante $(95,7 \%$ ). Conclusões: Verifica-se a necessidade de sensibilização da Atenção Primária, bem como o (re)conhecimento de quando o teste deve ser solicitado para oportunizar o início imediato do tratamento.

DESCRITORES: Tuberculose; Reação em Cadeia da Polimerase; Diagnóstico.

\section{ABSTRACT}

Objective: Describe the usability of the Rapid Molecular Test for Tuberculosis (TMR-TB) in Porto Velho, Rondônia. Method: Descriptive, cross-sectional and quantitative study, developed from the records of tuberculosis patients notified between January and August 2017 and who underwent TMR-TB. The data were analyzed using descriptive statistics, after meeting the ethical precepts. Results: 2,317 samples were analyzed, mainly requested by the penitentiary complex $(33.1 \%)$, the highest number of positive results in Tertiary Care $(27.8 \%)$, the highest number of requests in May $(19.6 \%)$ and positive results in June $(17.1 \%)$. The most analyzed sample was sputum $(97.6 \%)$, which represented $100 \%$ of positive results. The average time between diagnosis and start of treatment was 3.54 days $(\mathrm{SD}= \pm 8.32$ ), and the predominant pulmonary form $(95.7 \%)$. Conclusions: There is a need to raise awareness of Primary Care, and the recognition of when the test should be requested to provide an immediate start to treatment.

DESCRIPTORS: Tuberculosis; Polymerase Chain Reaction; Diagnosis.

\section{RESUMEN}

Objetivo Describir la usabilidad de la Prueba Molecular Rápida para la Tuberculosis (TMR-TB) en Porto Velho, Rondônia. Método: Estudio descriptivo, transversal y cuantitativo, desarrollado a partir de los registros de pacientes con tuberculosis notificados entre enero y agosto de 2017 sometidos a TMR-TB. Los datos fueron analizados mediante estadística descriptiva y cumplió los preceptos éticos. Resultado: Se analizaron 2.317 muestras, principalmente solicitadas por el complejo penitenciário (33,1\%), mayor cantidad de resultados positivos en Atención Terciaria (27,8\%), mayor número de solicitudes en mayo (19,6\%) y resultados positivos en junio $(17,1 \%)$. La muestra más analizada fue el esputo $(97,6 \%)$, representó el $100 \%$ de los resultados positivos. El tiempo medio entre el diagnóstico y el inicio del tratamiento fue de 3,54 días $(D E= \pm 8,32)$ y la forma pulmonar predominante $(95,7 \%)$. Conclusiones: Es necesario crear conciencia sobre la atención primaria, y reconocimiento de cuándo se debe solicitar la prueba para proporcionar un inicio inmediato del tratamiento.

DESCRIPTORES: Tuberculosis; Reacción en Cadena de la Polimerasa; Diagnóstico.

RECEBIDO EM: 20/02/2021 APROVADO EM: 08/03/2021 


\section{Rafaele Oliveira Bonfim}

Enfermeira do Serviço Social do Comércio de Rondônia, Mestre em Ciências da Saúde pelo Programa de Pós-graduação Enfermagem em Saúde Pública da Escola de Enfermagem de Ribeirão Preto da Universidade de São Paulo (EERP-USP).

ORCID: 0000-0001-8157-2323

\section{Caroline Lopes Vieira}

Enfermeira, Residência Multiprofissional em Saúde da Família pela Universidade Federal de Rondônia (UNIR). ORCID: 0000-0002-6984-7643

\section{Naime Oliveira Ramos \\ Enfermeira pela Universidade Federal de Rondônia (UNIR). \\ ORCID: 0000-0003-0566-495X}

\section{Melisane Regina Lima Ferreira}

Especialista em Saúde Pública e Doutoranda no Programa de Pós-Graduação Enfermagem em Saúde Pública da Escola de Enfermagem de Ribeirão Preto da Universidade de São Paulo (EERP-USP).

ORCID: 0000-0003-1694-5124

\section{Tatiane Cabral Siqueira}

Enfermeira, Especialista em Saúde Pública pela Fundação Universidade Federal de Rondônia (UNIR).

ORCID: 0000-0002-3860-1261

\section{Nathalia Halax Orfão}

Professora Doutora em Saúde Pública do Departamento de Medicina da Universidade Federal de Rondônia (UNIR).

ORCID: 0002-8734-3393

\section{INTRODUÇÃO}

A tuberculose (TB) é uma doença infectocontagiosa que se propaga pelo ar por meio de aerossóis contendo bacilos expelidos por um doente e que, ao serem inalados por pessoas sadias, provocam a infecção tuberculosa e o risco de desenvolver a doença ${ }^{1}$. A TB é um importante problema de saúde pública e, em 2018, tornou-se a principal causa de óbito no mundo por doença infecciosa de agente único².

No Brasil, no ano de 2018, foram diagnosticados 72.788 casos novos, correspondendo a um coeficiente de incidência de 34,8 casos/100 mil habitantes. Rondônia, apresentou o terceiro maior número de casos novos na Região Norte do país (582) e a capital, Porto Velho, um elevado coeficiente de incidência $(59.5 / 100 \mathrm{mil} \mathrm{hab}$.) e mortalidadede (2,5/100 mil hab.), além de ser o terceiro município em maior percentual de abandono do país $(24,6 \%)^{3}$.

Neste sentido, realizar a busca de sintomáticos respiratórios (SR), sobretudo dos bacilíferos, é uma das medidas mais eficientes para interrupção da cadeia de transmissão e controle da TB, tendo em vista que o diagnóstico precoce e início imediato do tratamento reduz o número de bacilos transmitidos pelo doente ${ }^{4}$.

Em um estudo que avaliou o retardo do diagnóstico da $\mathrm{TB}$, os gestores referiram dificuldades estruturais, falta de qualificação e número insuficiente de profissionais ${ }^{5}$. Tais aspectos influenciaram aproximadamente $50 \%$ dos doentes de TB que precisaram procurar quatro vezes ou mais os serviços de saúde para ter o diagnóstico adequado, o qual na maioria das vezes foi realizado em hospitais até mesmo pela gravidade dos sinais e sintomas ${ }^{6}$.

Em 2010, frente à problemática relacionada à demora no diagnóstico, um novo método baseado na biologia molecular foi desenvolvido - o Teste Molecular Rápido para a TB (TMR-TB), no qual é feito uma ampliação dos ácidos nucléicos para a detecção do Mycobacterium tuberculosis ${ }^{7}$. Esse método é capaz de reduzir o tempo do diagnóstico e identificar a sensibilidade e resistência para a Rifampicina, a qual é uma das principais drogas de primeira linha para o tratamento da TB.

No Brasil, o TMR-TB foi inserido como piloto nas capitais de Manaus e Rio de Ja- neiro por possuírem as maiores incidências da doença na federação ( 93,8 casos $/ 100$ mil hab. 12 e 82 casos/100 mil hab., respectivamente) $)^{8}$. Por este método, identificou-se um aumento na taxa de detecção bacteriológica de $\mathrm{TB}^{9}$, em tempo hábil de duas horas. $\mathrm{O}$ uso deste teste, antes da cultura ou baciloscopia, deve significar rapidez na elucidação diagnóstica ${ }^{7}$, sem desconsiderar a importância da avalição clínica pelo profissional de saúde. O intuito é promover o diagnóstico oportuno por meio do TMR-TB para os casos novos e utilizar a baciloscopia de escarro para os retratamentos (recidiva e reingresso após abandono) e controle mensal do tratamento.

A avaliação do TMR-TB constitui-se de uma importante ferramenta para contribuir na melhoria do diagnóstico e análise do desempenho dos serviços de saúde, uma vez que por meio desta, permite a identificação das fragilidades na oferta das ações, serviços e cuidado, além da observação das possíveis necessidades de capacitação ${ }^{10,11}$.

Em 2014, após a sua implementação, houve a distribuição de algumas máquinas pelo Ministério da Saúde para os estados, dentre eles Rondônia, a qual foi alocada 
no município de Porto Velho. Diante disso, este estudo visa descrever a usabilidade do TMR-TB para o diagnóstico da doença em Porto Velho-RO.

\section{MÉTODOS}

Trata-se de um estudo descritivo, realizado de forma transversal a partir de abordagem quantitativa, desenvolvido em Porto Velho, capital de Rondônia. Estima-se que a população do estado no ano de 2017 era de 1.805.788 habitantes e do município de 519.436 habitantes $^{12}$.

A atenção à TB é descentralizada para Atenção Primária à Saúde (APS), a qual é a porta de entrada para os SR, e deve realizar a solicitação dos exames e elucidação diagnóstica, acompanhamento dos casos de TB pulmonar, realização do Tratamento Diretamente Observado (TDO), investigação dos contatos e encaminhar para outros serviços de saúde, se necessário.

O município conta com o suporte de serviços especializados, em nível ambulatorial, que realizam o acompanhamento dos casos coinfectados TB/HIV, forma clínica extrapulmonar associada ou não a outras patologias, além dos casos de TB infantil. Em nível terciário, é realizado acompanhamento dos doentes de TB que apresentam resistência medicamentosa e internação, se necessário. Além disso, possui como suporte um laboratório municipal para a realização do TMR-TB e baciloscopia de escarro, bem como um laboratório estadual para cultura de escarro e teste de sensibilidade.

O resultado do TMR-TB é disponibilizado no Sistema Integrado de Informatiza-

Quadro 1. Levantamento das variáveis deste estudo, de acordo com a fonte de coleta de dados.

\begin{tabular}{|l|l|}
\hline FONTE DE COLETA DE DADOS & \multicolumn{1}{c|}{ VARIÁVEIS } \\
\hline Livro de Registro do TMR-TB & $\begin{array}{l}\text { Unidade de Saúde solicitante do TMR-TB } \\
\text { Resultado do TMR-TB } \\
\text { Tipo de amostra }\end{array}$ \\
\hline SINAN* & $\begin{array}{l}\text { Data do diagnóstico } \\
\text { Data do início do tratamento } \\
\text { Forma clínica da TB }\end{array}$ \\
\hline Elaborado pelas autoras. & \\
\hline
\end{tabular}

ção do Ambiente Hospitalar (HOSPUB) para a unidade solicitante do exame em até 48 horas. É importante que independentemente do resultado, seja solicitado o exame de cultura de escarro para investigação de micobactérias não causadoras de TB (MNT), conforme protocolo municipal.

A população do estudo foi constituída pelos SR que realizaram o TMR-TB no período de 12 de janeiro a 15 de agosto de 2017, totalizando 2.317 amostras para serem analisadas pelo teste. Foram excluídas 45 amostras da coleta de dados, sendo 10 por duplicidade de informação, 20 por ausência de kit (30 de maio de 2017), oito pela caracterização insatisfatória da amostra, seis por erro de solicitação do tipo de exame, tendo em vista que o TMR-TB é realizado apenas para diagnóstico, e a baciloscopia de escarro para controle mensal do tratamento, além de uma por descarte no Gerenciador de Ambiente Laboratorial (GAL) por se tratar de material para cultura.

Os dados foram coletados pelas pesquisadoras, por meio de um levantamento das variáveis no Livro de Registro do TMR-TB do laboratório municipal, o qual é alimentado na entrada das amostras para análise pelos profissionais que atuam no local e constam informações sobre o registro específico do livro, número de cadastro do HOSPUB, unidade solicitante, nome do paciente, resultado do TMR-TB e tipo de amostra; bem como, informações no Sistema de Informação de Agravos de Notificação (SINAN) para a coleta dos dados referentes à data de diagnóstico, data de início do tratamento e forma clínica (Quadro 1).

O fechamento do censo mensal, consolidado das informações sobre a quantidade de testes realizados pelo TMR-TB, era realizado a cada 30 dias, tendo início entre os dias 12 a 15 de um mês e término no mesmo dia do mês posterior.

A coleta foi armazenada em um banco de dados e analisados por meio de estatística descritiva no programa Statistica 13.4, da TIBCO. Para melhor interpretação dos achados, categorizou-se a variável unidade solicitante em Complexo Penitenciário, APS (Unidade de Saúde da Família e Uni- 
dades Básicas de Saúde de Porto Velho), atenção secundária (Unidade de Pronto Atendimento, Centro de Atenção Psicossocial, Policlínicas e Centro de Especialidades Médicas) e atenção terciária (Maternidade

Municipal Mãe Esperança, Hospital de Base Dr. Ary Pinheiro, Hospital Infantil Cosme e Damião, Hospital Estadual Pronto Socorro João Paulo II e Centro de Medicina Tropical de Rondônia).

Tabela 1. Distribuição do quantitativo de amostras analisadas pelo TMR-TB, de acordo com a unidade solicitante e resultado, no período de 12 de janeiro a 15 de agosto de 2017, Porto Velho, Rondônia, Brasil.

\begin{tabular}{|c|c|c|c|c|c|c|c|c|}
\hline \multirow[t]{2}{*}{ UNIDADE SOLICITANTE } & \multicolumn{2}{|c|}{$\begin{array}{c}\text { RESULTADO } \\
\text { POSITIVO }\end{array}$} & \multicolumn{2}{|c|}{$\begin{array}{c}\text { RESULTADO } \\
\text { NEGATIVO }\end{array}$} & \multicolumn{2}{|c|}{ ERRO } & \multicolumn{2}{|c|}{ TOTAL } \\
\hline & $\mathrm{N}$ & $\%$ & $\mathrm{~N}$ & $\%$ & $\mathrm{~N}$ & $\%$ & $\mathrm{~N}$ & $\%$ \\
\hline Complexo Penitenciário & 52 & 22,2 & 713 & 34,3 & 1 & 16,7 & 766 & 33,1 \\
\hline Atenção Secundária & 52 & 22,2 & 586 & 28,2 & - & - & 638 & 27,5 \\
\hline APS & 61 & 26,1 & 481 & 23,2 & 1 & 16,7 & 543 & 23,4 \\
\hline Atenção Terciária & 65 & 27,8 & 212 & 10,2 & 2 & 33,3 & 279 & 12,0 \\
\hline Outros municípios & 1 & 0,4 & 20 & 1,0 & 2 & 33,3 & 23 & 1,0 \\
\hline Saúde Indígena & - & - & 18 & 0,9 & - & - & 18 & 0,8 \\
\hline Distritos de Porto Velho & 2 & 0,9 & 15 & 0,7 & - & - & 17 & 0,7 \\
\hline Porto Velho - Zona Rural & - & - & 14 & 0,7 & - & - & 14 & 0,6 \\
\hline Instituição Privada & - & - & 6 & 0,3 & - & - & 6 & 0,3 \\
\hline Instituição Filantrópica & - & - & 5 & 0,2 & - & - & 5 & 0,2 \\
\hline Laboratório Estadual & 1 & 0,4 & 4 & 0,2 & - & - & 5 & 0,2 \\
\hline Consultório de Rua & - & - & 2 & 0,1 & - & - & 2 & 0,1 \\
\hline Outros Estados & - & - & 1 & 0,1 & - & - & 1 & 0,05 \\
\hline Total & 234 & 10,1 & 2.077 & 89,6 & 6 & 0,3 & 2.317 & 100 \\
\hline $\begin{array}{l}\text { Fonte: Livro de Registro TMR-TB/ Pol } \\
\text { Legenda: APS (Unidade de Saúde da } \\
\text { dimento, Centro de Atenção Psicosso } \\
\text { Esperança, Hospital de Base Dr. Ary P }\end{array}$ & Rafa & . & 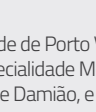 & & & 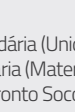 & ade 1 & $\begin{array}{l}\text { Aten- } \\
\text { at Mãe } \\
\text { III. }\end{array}$ \\
\hline
\end{tabular}

Tabela 2. Análise dos resultados mensais do TMR-TB, no período de 12 de janeiro a 15 de agosto de 2017, Porto Velho, Rondônia, Brasil

\begin{tabular}{lccccccccc} 
& \multicolumn{2}{c}{ MÊS } & \multicolumn{2}{c}{ POSITIVO } & \multicolumn{2}{c}{ NEGATIVO } & \multicolumn{2}{c}{ ERRO } & \multicolumn{2}{c}{ TOTAL } \\
& N & $\%$ & N & $\%$ & N & $\%$ & N & $\%$ \\
Janeiro & 21 & 9,0 & 127 & 6,1 & - & - & 148 & 6,4 \\
Fevereiro & 25 & 10,7 & 220 & 10,6 & 2 & 33,3 & 247 & 10,7 \\
Março & 37 & 15,8 & 264 & 12,7 & 1 & 16,7 & 302 & 13,0 \\
Abril & 34 & 14,4 & 286 & 13,8 & - & - & 320 & 13,8 \\
Maio & 36 & 15,4 & 416 & 20,0 & 1 & 16,7 & 453 & 19,5 \\
Junho & 40 & 17,1 & 324 & 15,6 & 2 & 33,3 & 366 & 15,8 \\
Julho & 24 & 10,3 & 244 & 11,8 & - & - & 268 & 11,6 \\
Agosto & 17 & 7,3 & 196 & 9,4 & - & - & 213 & 9,2 \\
Total & 234 & 10,1 & 2077 & 89,6 & 6 & 0,3 & 2.317 & 100 \\
Fonte: Livro de Registro TMR-TB/ Policlinica Rafael Vaz e Silva, 2017 & & & & & &
\end{tabular}

Além disso, no que concerne aos resultados das amostras (positivo, negativo e erro), aqueles que foram classificados como positivos (positivo/erro, resistentes e/ ou sensíveis à rifampicina) e os negativos (negativo/erro e negativo/encaminhado para cultura).

Este estudo atendeu os preceitos éticos previstos na Resolução 466/12 do Conselho Nacional de Saúde ${ }^{13}$, por meio do projeto matriz intitulado "Avaliação da situação epidemiológica da tuberculose no município de Porto Velho - RO" aprovado pelo Comitê de Ética em Pesquisa da Universidade Federal de Rondônia, conforme parecer 2.399.327 CEP/NUSAU/UNIR.

\section{RESULTADOS}

Verificou-se que o complexo penitenciário foi a unidade que mais solicitou o TMR-TB $(33,1 \%)$, seguido da atenção secundária $(27,5 \%)$, APS $(23,4 \%)$ e atenção terciária (12,0\%). Além disso, observou-se que a atenção terciária $(27,8 \%)$ e a APS $(26,1 \%)$ foram os níveis de atenção que obtiveram maior quantidade de resultados positivos (Tabela 1).

Além disso, no mês de maio ocorreu maior número de solicitações do TMR-TB (19,6\%), entretanto em junho houve maior percentual de resultado positivo $(17,1 \%)$ (Tabela 2).

Quanto ao tipo de material analisado, a maioria foi proveniente de amostra de escarro (97,6\%), porém ressalta-se que durante o período estudado houve análise de outros tipos de amostras, tais como líquor $(1,1 \%)$ e lavado gástrico e brônquico $(0,5 \%)$. Em relação ao resultado de amostra, foi possível identificar que todos os resultados positivos $(10,3 \%)$ foram das amostras de escarro (Tabela 3).

Observou-se que o tempo médio entre o diagnóstico e início do tratamento foi 3,54 dias ( $\mathrm{d} \mathrm{p}= \pm 8,32)$, sendo o mínimo de 0 dias e máximo de 61 dias, na qual independentemente da forma clínica, a maioria dos casos iniciou o tratamento no mesmo dia do diagnóstico do TMR-TB e houve predomínio da TB pulmonar (95,7\%) (Tabela 4). 


\section{artigo}

Bonfim, R.O.; Vieira, C.L.; Ramos, N.O.; Ferreira, M.R.L.; Siqueira, R.C.; Orfão, N.H.;

A usabilidade do teste molecular rápido para o diagnóstico da tuberculose em um município da amazônia ocidental

Tabela 3. Análise da quantidade e tipo de amostra solicitada mensalmente do TMR-TB, no período de 12 de janeiro a 15 de agosto de 2017, Porto Velho, Rondônia, Brasil

\begin{tabular}{|c|c|c|c|c|c|c|c|c|c|c|c|c|c|c|c|c|}
\hline \multirow{2}{*}{ MÊS } & \multicolumn{2}{|c|}{ ESC } & \multicolumn{2}{|c|}{ LíQ } & \multicolumn{2}{|c|}{ LAV } & \multicolumn{2}{|c|}{ URI } & \multicolumn{2}{|c|}{ ASP } & \multicolumn{2}{|c|}{ SAN } & \multicolumn{2}{|c|}{ Lín } & \multicolumn{2}{|c|}{ TOTAL } \\
\hline & N & $\%$ & $\mathrm{~N}$ & $\%$ & $\mathrm{~N}$ & $\%$ & $\mathrm{~N}$ & $\%$ & $\mathrm{~N}$ & $\%$ & $\mathrm{~N}$ & $\%$ & $\mathrm{~N}$ & $\%$ & $\mathrm{~N}$ & $\%$ \\
\hline Janeiro & 147 & 6,5 & - & - & 1 & 9,1 & - & - & - & - & - & - & - & - & 148 & 6,4 \\
\hline Fevereiro & 241 & 10,6 & 3 & 11,5 & 1 & 9,1 & 1 & 12,5 & 1 & 14,3 & - & - & - & - & 247 & 10,7 \\
\hline Março & 293 & 13,0 & 5 & 19,2 & 1 & 9,1 & 2 & 25,0 & - & - & 1 & 33,3 & - & - & 302 & 13,0 \\
\hline Abril & 309 & 13,7 & 7 & 26,9 & - & - & 1 & 12,5 & - & - & 2 & 66,7 & 1 & 100 & 320 & 13,8 \\
\hline Maio & 445 & 19,7 & 2 & 7,7 & - & - & 2 & 25,0 & 4 & 57,1 & - & - & - & - & 453 & 19,6 \\
\hline Junho & 353 & 15,6 & 2 & 7,7 & 7 & 63,6 & 2 & 25,0 & 2 & 28,6 & - & - & - & - & 366 & 15,8 \\
\hline Julho & 264 & 11,7 & 4 & 15,4 & - & - & - & - & - & - & - & - & - & - & 268 & 11,6 \\
\hline Agosto & 209 & 9,2 & 3 & 11,6 & 1 & 9,1 & & - & - & - & & - & & - & 213 & 9,2 \\
\hline \multicolumn{17}{|c|}{ RESULTADO DA AMOSTRA } \\
\hline Positivo & 234 & 10,3 & - & - & - & - & - & - & - & - & - & - & - & - & 234 & 10,1 \\
\hline Negativo & 2021 & 89,4 & 26 & 100 & 11 & 100 & 8 & 100 & 7 & 100 & 3 & 100 & 1 & 100 & 2077 & 89,6 \\
\hline Erro & 6 & 0,3 & - & - & - & - & - & - & - & - & - & - & - & - & 6 & 0,3 \\
\hline Total & 2261 & 97,6 & 26 & 1,1 & 11 & 0,5 & 8 & 0,35 & 7 & 0,3 & 3 & 0,1 & 1 & 0,05 & 2317 & 100 \\
\hline
\end{tabular}

Tabela 4. Análise do tempo entre o diagnóstico da TB pelo TMR-TB e o início do tratamento, de acordo com a forma clínica da doença, no período de 12 de janeiro a 15 de agosto de 2017, Porto Velho, Rondônia, Brasil.

\begin{tabular}{|c|c|c|c|c|c|c|c|c|}
\hline \multirow{3}{*}{$\begin{array}{l}\text { TEMPO PARA } \\
\text { O INÍCIO DO } \\
\text { TRATAMENTO }\end{array}$} & \multicolumn{6}{|c|}{ FORMA CLÍNICA } & \multirow{2}{*}{\multicolumn{2}{|c|}{ TOTAL }} \\
\hline & \multicolumn{2}{|c|}{ PULMONAR } & \multicolumn{2}{|c|}{ EXTRAPULMONAR } & \multicolumn{2}{|c|}{$\begin{array}{l}\text { PULMONAR + } \\
\text { EXTRAPULMONAR }\end{array}$} & & \\
\hline & $\mathbf{N}$ & $\%$ & $\mathbf{N}$ & $\%$ & $\mathbf{N}$ & $\%$ & $\mathbf{N}$ & $\%$ \\
\hline 0 dias & 106 & 60,2 & 6 & 100 & 1 & 50,0 & 113 & 61,4 \\
\hline 1 a 2 dias & 26 & 14,8 & - & - & 1 & 50,0 & 27 & 14,7 \\
\hline 3 a 7 dias & 14 & 8,0 & - & - & - & - & 14 & 7,6 \\
\hline 8 a 14 dias & 16 & 9,1 & - & - & - & - & 16 & 8,7 \\
\hline 15 a 30 dias & 11 & 6,2 & - & - & - & - & 11 & 6,0 \\
\hline$>30$ dias & 3 & 1,7 & - & - & - & - & 3 & 1,6 \\
\hline Total & 176 & 95,6 & 6 & 3,3 & 2 & 1,1 & 184 & 100 \\
\hline Fonte: SINAN, 2017. & & & & & & & & \\
\hline
\end{tabular}

\section{DISCUSSÃO}

Diante dos resultados encontrados, verificou-se que embora tenha ocorrido a usabilidade do TMR-TB para o diagnóstico da doença, a cobertura pelo teste foi baixa, visto o número de habitantes do estado e a quantidade de testes realizados durante o período do estudo. Ao mesmo tempo em que, tal quantitativo foi realizado apenas em uma máquina, a qual estava disponível para todo o estado, e poderia não conseguir suprir a demanda. Este cenário pode ser alterado com a aquisição, em novembro de 2017, das novas máquinas pela Agência Estadual de Vigilância em Saúde (AGEVISA) pertencente à Secretaria de Estado da Saúde de Rondônia, instaladas quatro no interior em municípios sedes das Regiões de Saúde e mais uma na capital no serviço de referência estadual terciária ${ }^{14}$.

Ainda que o número de testes solicitados pelo Complexo Penitenciário tenha sido maior quando comparado com as outras unidades de saúde, está bem aquém do preconizado, uma vez que Rondônia possui 53 unidades prisionais, com população carcerária de 7.631 indivíduos, mesmo com 4.928 vagas $^{15}$.

Tal achado reflete nas fragilidades da baixa busca ativa e rastreamento da TB mesmo diante do aumento das pessoas privadas de liberdade (PPL), superlotação, pouca circulação de ar nas celas, alta rotatividade da PPL nos centros de detenção, e compartilhamento do mesmo local entre aqueles que aguardam julgamento com os já condenados, o que torna as instalações inadequadas e um ambiente propício para a proliferação das doenças infectocontagiosas 16, e consequentemente, aumenta a susceptibilidade à infecção dentro da PPL, familiares e funcionários ${ }^{17,18}$. Isto pode levar ao agravamento da saúde dessa população, resistência às medicações e até mesmo ao óbito. 
Outros desafios são a morosidade na identificação dos casos suspeitos de TB, dificuldades da PPL em relação ao início e continuidade do tratamento após o diagnóstico nas unidades prisionais e fora delas para aqueles que estão no regime semiaberto $^{19}$, bem como a falta de profissionais para dar segmento ao TDO, sendo que em alguns casos, são os próprios agentes penitenciários que o realizam.

No que concerne à atenção secundária, tal achado pode refletir na dinâmica funcional das instituições, que limitam as ações de controle e levam ao retardo ao diagnóstico, seja na detecção dos SR e/ ou demora na confirmação diagnóstica, falta de conhecimento e/ ou sensibilização dos profissionais diante de um caso suspeito, acessibilidade e horário de funcionamento da $\mathrm{APS}^{19,20}$, além da dificuldade na forma como os serviços de saúde se organizam para diagnosticar e detectar precocemente os $\operatorname{casos}^{21}$.

Nota-se que a atenção à TB na APS é fragilizada, na medida em que muitas vezes os profissionais desconhecem o número de casos no seu território e, em outros momentos, omitem a sua responsabilidade no acompanhamento e ações de controle da TB, diante das reais necessidades e vulnerabilidades da população adscrita ${ }^{7,22}$.

Os diversos métodos laboratoriais estão disponíveis para a detecção, contudo, os profissionais precisam encontrar saídas para o diagnóstico oportuno. O TMR$-\mathrm{TB}$, neste caso, especificamente, é um instrumento para a confirmação dos casos e identificação da sensibilidade e resistência à rifampicina em curto prazo quando comparado com a baciloscopia e cultura de escarro, respectivamente.

Entretanto, como o sujeito compreende-se doente também deve ser discutido, uma vez que as fragilidades no acesso e resolução dos seus problemas na APS contribuem no retardo do diagnóstico, o qual por inúmeras vezes é realizado em outros níveis de atenção, conforme ocorreu neste estudo com o maior quantitativo de resultados positivos na Atenção Terciária. Tal achado corrobora com um estudo realizado em Porto Velho que identificou que a elucidação diagnóstica não tem ocorrido

\section{Os diversos métodos}

laboratoriais estão

disponíveis para a

detecção, contudo,

os profissionais

precisam encontrar

saídas para o

diagnóstico

oportuno. O TMR-

\section{TB, neste caso,}

especificamente, é

um instrumento para

a confirmação dos

casos e identificação

da sensibilidade

e resistência à

rifampicina em

curto prazo quando

comparado com

a baciloscopia e

cultura de escarro,

respectivamente. na APS, ressaltando a baixa resolutividade do nível primário e as fragilidades locais para ações do acompanhamento e controle da TB, dentre eles a articulação entre os serviços de saúde ${ }^{23}$.

A percepção do sujeito no processo saúde-doença é subjetiva, considerando as singularidades inerentes ao indivíduo, bem como sua situação socioeconômica e cultural, e ao meio em que este está inseri$\mathrm{do}^{24}$. Além disso, alguns sintomas pontuais, tais como tosse, febre e emagrecimento quando não impedem os SR de exercerem suas atividades diárias, acabam sendo negligenciados, afetando na busca imediata por atendimento.

A APS deveria ser a primeira unidade, como porta de entrada, a solicitar o TMR-TB, tendo em vista o seu papel de coordenadora do cuidado e ordenadora da Rede de Atenção à Saúde, visando à promoção e prevenção da saúde, e, quando necessário, referenciar para outro nível de atenção $0^{25,26}$.

A acessibilidade pode ser entendida como o grau de ajuste às características da oferta do serviço e da população, incluindo o processo de busca e a obtenção da assistência, portanto, a escolha da unidade está ligada com as experiências anteriores, facilidade de acesso, garantia de atendimento e resolutividade do problema de saúde, no qual os níveis de assistência precisam estar preparados para estas demandas, a fim de garantir que os pontos de atenção considerados prioritários e sensíveis para $\mathrm{TB}$ sejam resolutivos ${ }^{27}$.

Estudos abordam que os usuários procuram resolutividade na atenção secundária e/ ou terciária, bem como em unidades de saúde fora de sua área de abrangência, seja pela percepção da sua relação com a oferta do serviço, densidade tecnológica ou até mesmo pelo próprio estigma da doença ${ }^{19,20,26}$. Neste sentido, ressalta-se a importância das capacitações para sensibilizar e auxiliar os profissionais para realizarem o diagnóstico precoce para a TB, além do desenvolvimento de ações de educação em saúde com a comunidade.

Observou-se ainda que o maior número de solicitações do TMR-TB aconteceu em maio. Sabe-se que a Região Norte pos- 


\section{artigo}

Bonfim, R.O.; Vieira, C.L.; Ramos, N.O.; Ferreira, M.R.L.; Siqueira, R.C.; Orfão, N.H.;

A usabilidade do teste molecular rápido para o diagnóstico da tuberculose em um município da amazônia ocidental

sui um período chuvoso e com baixa temperatura que ocorre entre o fim de novembro e início de abril, no qual a população tende a se manter mais tempo em ambientes fechados, facilitando a proliferação e infecção pelo bacilo. Logo, percebe-se que pode existir uma sazonalidade da TB para o aumento de solicitações no mês de maio no município estudado.

Destaca-se que o maior número de casos positivos foi proveniente das amostras de escarro, refletindo a forma clínica predominante, a pulmonar. O TMR-TB possui maior sensibilidade quando se trata de uma amostra de escarro, não necessitando de grandes quantidades de bacilos para isso. Tal achado vai de encontro com a literatura atual que apontam que as amostras de escarro, quando satisfatórias, são facilmente detectadas pelo TMR-TB ${ }^{28}$.

A predominância da forma clínica pulmonar justifica-se ao fato de que o bacilo cresce em local oxigenado e com grande fluxo sanguíneo. A TB pulmonar é um importante problema de saúde pública e necessita de métodos para que seja controlada, considerando suas implicaçõos ${ }^{29}$, pois trata-se de uma das formas transmissíveis da doença, sendo necessário o diagnóstico oportuno por meio do TMR-TB, e início imediato do tratamento, visto as vantagens de análise e resultado para detecção do bacilo no teste quando compa- rado a outros métodos convencionais de diagnóstico, ${ }^{90}$.

Contudo, observou-se que mesmo que a maioria dos doentes de TB tenha iniciado a terapêutica medicamentosa no mesmo dia, um número significativo de indivíduos começou tardiamente. Esse achado é preocupante, pois além das implicações que esse retardo pode ocasionar quanto à continuidade da cadeia de transmissão, traz consigo os agravos relacionados à condição de saúde do indivíduo mesmo com o diagnóstico precoce da $\mathrm{TB}^{30}$.

Ao mesmo tempo em que outros fatores poderiam contribuir na demora, a exemplo da organização dos serviços, fragilidades no sistema de informatização e na busca dos SR. Ressalta-se também a ausência de estrutura adequada na APS, facilidade no acesso físico, financeiro, geográfico e estrutural para fornecer uma Rede de Atenção à Saúde integrada e articulada ${ }^{26}$.

O TMR-TB disponibiliza o resultado de quatro amostras analisadas, concomitantemente, confirmando (ou não) o caso em duas horas. Contudo, o cenário local, no período deste estudo, possuía uma única máquina para a análise das solicitações provenientes dos diferentes níveis de atenção do estado, o que poderia acarretar em uma fila de espera, retardo na análise das amostras e disponibilização dos resulta- dos, mas que não influenciaria no início do tratamento.

\section{CONCLUSÃO}

Os resultados encontrados neste estudo por meio da avaliação da usabilidade do TMR-TB revelam o seu caráter inovador, considerando a importância para o diagnóstico da doença e o benefício que esta nova tecnologia em saúde trouxe para o estado e para o município em relação ao diagnóstico da doença e, medidas de controle do agravo, principalmente diante da situação epidemiológica da TB e das MNT.

Neste sentido, é essencial que haja sensibilização dos gestores e profissionais da APS para a utilização do TMR-TB, com ações voltadas para vigilância em saúde, estratégias para a corresponsabilização da população no controle da TB e reorganização da APS para o fortalecimento deste nível de atenção por meio de um planejamento articulado entre o Programa de Controle da Tuberculose e a coordenação do Departamento da Atenção Básica.

Como limitação deste estudo, ressalta-se a não informatização dos registros acerca das solicitações do TMR-TB, a qual poderia contribuir na avaliação local, em tempo real, nas unidades de saúde e gestão.

\section{REFERÊNCIAS}

1. Souza EP, Barbosa ECS, Rodrigues ILA. Prevenção e controle da tuberculose: revisão integrativa de literatura. Revista Cuidarte, v. 6, n. 2, p. 1093-101, 2015. DOI: doi.org/10.1590/ S0080-623420130000600018.

2. World Health Organization. Global tuberculosis report 2019. Geneva: World Health Organization; 2019.

3. Brasil. Ministério da Saúde. Secretaria de Vigilância em Saúde. Boletim Epidemiológico Tuberculose. Brasil livre da tuberculose: evolução dos cenários epidemiológicos e operacionais. 2019. Secretaria de Vigilância em Saúde. Brasília, DF, v. 50, 2020.

4. Silva DA, Yamamura M, Figueiredo RM. Tuberculose no grupo de vigilância epidemiológica (GVE) XII - Araraquara: incidência e perfil das notificações de 2009 a 2013. Revista CuidArte Enfermagem, v. 10, n. 1, p. 15-21, 2016. Disponivel em: http:// fundacaopadrealbino.org.br/facfipa/ner/pdf/CuidarteEnferma-
gemvolume10Jan- Jun2016.pdf.

5. Sá LD, Barrêto AJR, Nogueira JA, Cunha FTS, Palha PF, Villa TCS. A discursividade de gestores sobre aspectos relacionados ao retardo do diagnóstico de tuberculose. Rev Esc Enferm USP, v. 47, n. 5, p. 1165-1171, 2013. DOI: doi.org/10.1590/S0080623420130000500022 .

6. Ponce MAZ, Wysocki AD, Arakawa T, Andrade RLP, Vendramini SHF, Sobrinho RAS, Monroe AA, Netto AR, Villa TCS. Atraso do diagnóstico da tuberculose em adultos em um município paulista em 2009: estudo transversal. Epidemiol. Serv. Saúde, v. 25, n. 3, p. 552- 562, 2016. DOI: doi.org/10.5123/S167949742016000300011.

7. Andrade DFR, Carvalho ML, Araújo ME, Sousa MAS, Sá LC, Araújo EJB. Vantagens do teste rápido molecular para tuberculose: uma revisão integrativa. Rev. Enf. UFSM v. 7, n. 1, p. 123- 


\section{REFERÊNCIAS}

135, 2017. DOI: doi.org/10.5902/2179769221894.

8. BRASIL. Ministério da Saúde. Secretaria de Vigilância em Saúde. Departamento de Vigilância em Doenças Transmissíveis. Rede de Teste Rápido para Tuberculose no Brasil: primeiro ano de implantação [Internet]. 2015 [acessado em 29 out 2020]. Disponivel em: http://portalarquivos.saude.gov.br/images/ pdf/2016/janeiro/19/rtr-tb-15jan16-isbn-web.pdf.

9. BRASIL. Ministério da Saúde. Boletim Brasileiro de Avaliação de Tecnologia em Saúde [Internet]. 2011 [acessado em 29 out 2020]. Disponivel em: hhttp://bvsms.saude.gov.br/bvs/periodicos/brats_16.pdf.

10. Silva DM, Nogueira JA, Sá LD, Wysocki AD, Scatena LM, Villa TCS. Avaliação de desempenho de serviços de atenção básica para o tratamento da tuberculose. Rev Esc Enferm USP, v. 48 , n. 6 , p. $1044-53,2014$. DOI: doi.org/10.1590/S0080623420140000700012 .

11. Sieiro TLA, Aurílio RB, Soares ECC, Chiang SS, Sant'Anna CC. The role of Xpert MTB/RIF essay among adolescents suspected of pulmonary tuberculosis in Rio de Janeiro, Brazil. Rev. Soc Bras. Med. Trop., v. 51, n. 2, p. 234-36, 2018. DOI: doi: 10.1590/00378682-0298-2017.

12. Instituto Brasileiro de Geografia e Estatística. Características da Estimativas populacionais para os municípios e para as Unidades da Federação brasileiros em 01.07.2017 [Internet]. IBGE; 2017 [acessado em 23 ago 2017] Disponivel em: ftp://ftp.ibge. gov.br/Estimativas_de_Populacao/Estimativas_2017/estimativa_dou_2017.pdf

13. Brasil. Ministério da Saúde. Resolução № 466, de 12 de dezembro de 2012. Brasília. 2012.

14. Rondônia. Secretaria do Estado da Saúde. Diagnóstico e início do tratamento contra tuberculose pode começar em apenas duas horas em Rondônia, anuncia Sesau. Porto Velho, 2017. Disponível em: <http://www.rondonia.ro.gov.br/diagnostico-e-inicio-do-tratamento- contra-tuberculose-pode-comecar-em-apenas-duas-horas-em-rondonia-anuncia-sesau/>. Acesso em: 28 de nov. de 2017.

15. Brasil. Ministério da Justiça. Levantamento Nacional de Informações Penitenciária - INFOPEN. Departamento Penitenciário Nacional [Internet], Brasília, DF, 2014. Disponível em: http://dados.mj.gov.br/dataset/infopen-levantamento-nacional-de-informacoes-penitenciarias.

16. Larouzé B, Ventura M, Sánchez AR. Tuberculose nos presídios brasileiros: entre a responsabilização estatal e a dupla penalização dos detentos. Cad. Saúde Pública, v. 31, n. 6, p $1127-$ 1130, 2015. DOI: doi.org/10.1590/0102-311XPE010615.

17. Sacramento DS, Gonçalves MJF. Situação da tuberculose em pessoas privadas de liberdade no período de 2007 a 2012. Rev Enf UFPE, v. 11, n. 1, p. 140- 150, 2017. DOI: doi.org/10.5205/ reuol.9978-88449-6-1101201717.

18. Allgayer MF, Ely KZ, Freitas GH, Valim ARM, Krug SBF, Possuelo LG. Tuberculose: vigilância e assistência à saúde em prisões. Rev. Bras. Enferm., v. 72, n. 5, p. 1370-1377, 2019. DOI: doi.org/10.1590/0034-7167-2018-0260.

19. Brunello MEF, Orfão NH, Andrade RLP, Magnabosco GT, Arakawa T, Monroe AA, Silva-Sobrinho RA, Netto AR, Villa TCS. et al. Tuberculose: análise do percurso do doente durante o tratamento em Ribeirão Preto/SP. Rev. Eletr Enf., v. 18, e1176, p. 1-12, 2016. DOI: doi.org/10.5216/ree.v18.39051.

20. Macedo LR, Maciel ELN, Struchiner CJ. Fatores associados à tuberculose na população privada de liberdade no Espírito Santo. Rev. Saúde Pública, v. 54, n. 67, p. 1-12, 2020. DOI:doi. org/10.11606/s1518-8787.2020054001818.

21. Orfão NH, Crepaldi NY, Brunello MEF, Andrade RLP, Monroe AA, Netto AR, Villa, TCS. Coordenação da assistência à tuberculose: registro de dados e a implementação de um sistema informatizado. Ciênc. Saúde Coletiva, v. 22, n. 6, p. 1969-1977, 2017. DOI: doi.org/10.1590/1413-81232017226.15352016.

22. Oliveira LCS, Nogueira JA, Sá LD, Palha PF, Silva CA, Villa TCS. A discursividade do sujeito sobre sentimentos associados ao enfrentamento da tuberculose. Rev. Eletr Enf., v. 7, n. 1, p. 12-20, 2015. DOI: doi.org/10.5216/ree.v17i1.24523.

23. Silva LT, Felipini MC, Oliveira TB, Brunello MEF, Orfão NH. Perfil epidemiológico da tuberculose no serviço de referência do estado de Rondônia. Rev. Epidemiol. Controle Infecç., v. 9, n. 1, p. 1-7, 2019. DOl: doi.org/10.17058/reci.v9i1.12249.

24. Starfield, B. Atenção Primária: equilíbrio entre necessidades de saúde, serviços e tecnologia. Brasília: UNESCO, Ministério da Saúde, 2002. 726 p. Disponível em: http://www.dominiopublico.gov.br/pesquisa/DetalheObraForm.do?select_action=\&co_obra=14609.

25. Mendes EV. As redes de atenção à saúde. $2^{a}$ ed. Brasília: Organização Pan-Americana da Saúde; 2011.

26. Martins MDR, Valerão NB, Tomberg JO, Spagnolo LML, Soares LN, Gonzales RIC. Serviço de saúde procurado pelas pessoas com sintomas de tuberculose. Rev. Enferm. UFSM, v. 9, e22, p. 1-16, 2019. DOI: doi.org/10.5902/217976923049.

27. Habte D, Melese M, Hiruy N, Gashu Z, Jerene D, Moges F, Yifru S, Girma B, Kassie Y, Haile YK, Suarez PG, Tessema B. The additional yield of GeneXpert MTB/RIF test in diagnosis of pulmonary tuberculosis among household contacts of smear positive TB cases. International Journal of Infesctious Diseases, v. 49, p. 179-184, 2016. DOI: doi.org/10.1016/j.jijid.2016.07.002.

28. Oliveira MG, Jomar RT, Motta MCS. Tuberculose pulmonar entre usuários de uma Unidade de Atenção Básica. Rev. Bras. Promoç. Saúde, v. 28, n. 1, p. 106-112, 2015. DOI: doi. org/10.5020/18061230.2015.p106.

29. Lima TM, Belotti NCU, Nardi SMT, Pedro HSP. Teste rápido molecular GeneXpert MTB/RIF para diagnóstico da tuberculose. Rev Pan-Amaz Saude, v. 8, n. 2, p. 67-78, 2017. DOl: doi. org/10.5123/S2176- 62232017000200008.

30. Kwak N, Choi SM, Lee J, Park YS, Lee C, Lee S, Yoo C, Kim YW, Han SK, Yim J. Diagnostic accuracy and turnaround time of the Xpert MTB/RIF assay in routine clinical practice. Plos one, v. 8, n. 10, p. 1-15, 2013. DOI: doi.org/10.1371/journal.pone.0077456. 